\title{
Prevalence and public health relevance of micronutrient deficiencies and undernutrition in pre-school children and women of reproductive age in Côte d'lvoire, West Africa
}

\author{
Fabian Rohner ${ }^{1,2, *}$, Christine Northrop-Clewes ${ }^{2}$, Andres B Tschannen ${ }^{3}$, \\ Patrice E Bosso ${ }^{4}$, Valérie Kouassi-Gohou ${ }^{5}$, Juergen G Erhardt ${ }^{6}$, Mai Bui ${ }^{7}$, \\ Michael B Zimmermann ${ }^{8}$ and CG Nicholas Mascie-Taylor ${ }^{9}$ \\ 'GroundWork LLC, 40b Les Landes, 1299 Crans-près-Céligny, Switzerland: ${ }^{2}$ GAIN - Global Alliance for \\ Improved Nutrition, Geneva, Switzerland: ${ }^{3}$ Centre Suisse des Recherches Scientifiques, Abidjan, Côte d'Ivoire: \\ ${ }^{4}$ Helen Keller International Côte d'Ivoire, Abidjan, Côte d'Ivoire: ${ }^{5}$ Direction de I'Information de la Planification et \\ de l'Evaluation, Ministère de la Santé et de l'Hygiène Publique, Abidjan, Côte d'Ivoire: 'SEAMEO-TROPMED, \\ University of Jakarta, Jakarta, Indonesia: ${ }^{7}$ Swiss Vitamin Institute, Epalinges, Switzerland: ${ }^{8}$ Institute of Food \\ Science and Nutrition, ETH Zurich, Switzerland: ${ }^{9}$ Department of Biological Anthropology, University of \\ Cambridge, Cambridge, UK
}

Submitted 5 December 2012: Final revision received 5 July 2013: Accepted 23 July 2013: First published online 4 September 2013

\begin{abstract}
Objective: To provide nationally representative data on the prevalence of anaemia, vitamin A and Fe deficiencies among pre-school age children (pre-SAC) and nonpregnant women of reproductive age (WRA), and on vitamin $\mathrm{B}_{12}$ and folate deficiencies in WRA, and the influence of inflammation on their interpretation.

Design: A cross-sectional survey to measure anthropometry, malaria parasitaemia and micronutrient status. Specifically, blood samples were analysed for $\mathrm{Hb}$, plasma ferritin, soluble transferrin receptors, C-reactive protein, $\alpha_{1}$-acid glycoprotein, retinol-binding protein, vitamin $\mathrm{B}_{12}$ and folate.

Setting: Côte d'Ivoire in 2007.

Subjects: Nine hundred and twenty-eight WRA and 879 pre-SAC.

Results: In WRA, prevalence of Plasmodium parasitaemia (5\%) was low, but inflammation (34\%) was higher. Anaemia was a severe public health problem and prevalence differed by residency and eco-region. Inflammation-adjusted Fe deficiency was highest in urban areas (20\%). Nationally, folate deficiency was $86 \%$, higher in urban areas and varied by eco-region. Prevalence of vitamin $\mathrm{B}_{12}$ deficiency was low but higher in the rural areas and the north. Inflammationadjusted vitamin A deficiency was very low (1\%). In pre-SAC, prevalence of inflammation (67\%) and Plasmodium parasites (25\%) was high; the latter was associated with poverty, rural residency and higher ferritin concentrations. Anaemia was classified as a severe public health problem ( $72 \%)$, and was higher in rural areas $(76 \%)$ and the north $(87 \%)$. A quarter of pre-SAC suffered from vitamin A deficiency (inflammation-adjusted) and prevalence of undernutrition was high. Conclusions: Prevalence of inflammation, Plasmodium parasitaemia and micronutrient deficiencies were high in Côte d'Ivoire, particularly in pre-SAC. Nutritional interventions should be accompanied by strategies to reduce exposure to infections.
\end{abstract}

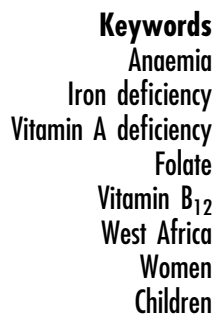

According to the WHO, anaemia affects $68 \%$ of preschool age children (pre-SAC) and $48 \%$ of non-pregnant women of reproductive age (WRA) in Africa ${ }^{(1)}$. The aetiology of anaemia in sub-Saharan Africa is multifactorial $^{(2)}$ and although Fe deficiency is probably the most common cause, it may also be associated with inflammation, acute and chronic infections ${ }^{(3)}$, other micronutrient deficiencies, especially of folate, vitamin $\mathrm{B}_{12}$ and vitamin $\mathrm{A}^{(4)}$, and genetically inherited $\mathrm{Hb}$ traits. The health and social consequences of anaemia and $\mathrm{Fe}$ deficiency are well characterized and include impaired cognition, poorer educational achievement, increased morbidity and mortality in children, and reduced work capacity and poor pregnancy outcomes in WRA ${ }^{(5,6)}$. Important strategies to combat anaemia include Fe supplementation, Fe fortification of staple foods, which has been shown to be efficacious and cost-effective ${ }^{(7)}$, and infection control to increase Fe uptake and absorption ${ }^{(8)}$.

In 1996, a survey conducted in Côte d'Ivoire covering four regions found anaemia in $50 \%$ and $42 \%$, and $\mathrm{Fe}$ 
deficiency in $63 \%$ and $41 \%$, of pre-SAC and WRA, respectively ${ }^{(9)}$. The Ministry of Health adopted a strategy to reduce anaemia including Fe supplementation, dietary counselling, food diversification, food fortification and public health measures to combat malaria and other parasitic diseases.

In 2005, the 'Communauté Economique des Etats de l'Afrique de l'Ouest' estimated that $42 \%$ of children were at risk of vitamin A deficiency (VAD) and adequate control could decrease the prevalence by $25 \%{ }^{(10)}$. A quasinational survey in Côte d'Ivoire covering three rural and one urban area reported severe VAD (serum retinol $<0.35 \mu \mathrm{mol} / \mathrm{l}$ ) in $24 \%$ and $7 \%$, and moderate VAD (serum retinol $<0.7 \mu \mathrm{mol} / \mathrm{l}$ ) in $56 \%$ and $21 \%$, of pre-SAC and WRA, respectively ${ }^{(11)}$. Diets are monotonous and based on cereals and legumes that are poor sources of vitamin A. VAD is found associated with low intake and/or absorption of vitamin A and with inflammation ${ }^{(12)}$ and was a public health concern in Côte d'Ivoire, but its magnitude seemed to vary across social and geographical strata. In 2007, the twice annual supplementation of pre-SAC with vitamin A capsules was not linked to the immunization programme ${ }^{(13)}$ and the postnatal supplementation of women was having limited reach. Fortification of vegetable oil with vitamin A was in place, and $50-70 \%$ of commercialized vegetable oil was estimated to be fortified in 2007 .

By 2007, the socio-economic situation in Côte d'Ivoire had worsened due to the internal conflict, and there were indications that nutritional and health status had deteriorated along with an increase in poverty ${ }^{(14)}$. Existing sub-national data on the prevalence of anaemia, vitamin $\mathrm{A}$ and $\mathrm{Fe}$ deficiencies were from the period before the conflict in Côte d'Ivoire and no literature on folate and vitamin $\mathrm{B}_{12}$ deficiencies was found. It was therefore proposed to carry out a representative cross-sectional survey in the nine eco-regions of Côte d'Ivoire (rural and urban), to obtain nationally representative data with the statistical power to compare eco-regions and to determine the prevalence of anaemia, vitamin $\mathrm{A}$ and $\mathrm{Fe}$ deficiencies and the influence of inflammation on their interpretation among pre-SAC and non-pregnant WRA, and the prevalence of vitamin $B_{12}$ and folate deficiencies in WRA.

\section{Materials and methods}

\section{Survey design and participant enrolment}

The design and participant enrolment procedure is described in detail elsewhere ${ }^{(15)}$. In brief, a nationally representative cross-sectional survey was conducted in Côte d'Ivoire in July/August 2007. The country was disaggregated into nine eco-regions, and the sampling strategy, using a proportionate-to-population size approach, was based on the general population census of $1998^{(16)}$. In total, there were sixty clusters with equal numbers from rural and urban areas. In each cluster, sixteen households were selected using the Expanded Programme on Immunization (EPI) method. Within the households, the study focused on children aged 6-59 months and non-pregnant (by self-declaration) WRA aged 15-49 years. Only one subject per age group was selected using the Kish table for random selection ${ }^{(17)}$, resulting in an expected sample size of 960 pre-SAC and 960 non-pregnant WRA.

After the household selection and consent to take part in the survey, all consenting and eligible subjects were registered (household, sex, age, date, ID number). The respondents answered questions on demographics, health and socio-economic status (housing quality, access to water, electricity and transport) and eligible household members were then invited to go to the nearest health facility to have anthropometric measurements taken and give a blood sample.

\section{Etbics and consent}

Approval for the study was granted by the Comite National d'Ethique des Sciences de la Vie et de la Santé, Abidjan, Côte d'Ivoire (clearance number 4027).

Inclusion in the survey was dependent on the household head being willing to participate. Written informed consent was also sought from the participating WRA or the parent or legal guardian of the participating child. Illiterate but consenting WRA or parents/guardians were asked to provide a fingerprint in lieu of a signature. Exclusion criteria included refusal to participate or the presence of medical contraindications for blood drawing.

\section{Antbropometry}

Anthropometric measurements were taken by trained field staff using standard anthropometric techniques. If children were $>2$ years of age, standing height measurement was done; for younger children their length was assessed. Weight was recorded to the nearest $0 \cdot 1 \mathrm{~kg}$ and height to the nearest $0.5 \mathrm{~cm}$.

\section{Blood sampling and analysis}

Intravenous blood samples were collected from an antecubital vein of WRA and children $>12$ months of age; for younger children blood was collected from the heel. The venous blood samples $(7.5 \mathrm{ml}$ from WRA, $2.5 \mathrm{ml}$ from children) were drawn into EDTA-treated evacuated tubes (Vacutainer; Becton Dickinson, Franklin Lakes, NJ, USA). Immediately after mixing, the $\mathrm{Hb}$ concentration was determined using a Hemocue photometer ( $\mathrm{Hb} 201+$; HemoCue AB, Angelholm, Sweden), the results noted and also reported to the respondent.

After phlebotomy and on-site diagnostics, the remaining whole blood was stored on ice and protected from direct light until further processing. Later the same day, malaria smears were prepared, then the whole blood was centrifuged and the plasma aliquoted and stored at $-25^{\circ} \mathrm{C}$ for later transportation and analysis. 
For analysis of $\mathrm{Hb}$ concentration, the Hemocue photometer was used. Normal and low level controls, provided by the supplier, were used to test the Hemocue twice daily.

After completion of a cluster, thick and thin blood films for malaria testing were stained with Giemsa and dried for storage. Malaria slides were examined under a microscope for species-specific Plasmodium infection by experienced technicians. Parasites were counted against 200 leukocytes (if fewer than ten parasites were identified, counting was continued up to 500 leukocytes). Counts were converted to the number of parasites/ $\mu$ l of blood, assuming a leukocyte count of $8000 / \mu l^{(18)}$.

Plasma was analysed for retinol-binding protein (RBP), ferritin, soluble transferrin receptor (STfR), C-reactive protein (CRP) and $\alpha_{1}$-acid glycoprotein (AGP) at the VitAIron Tech laboratories (Wilstaett, Germany), in one run, from $<100 \mu$ l plasma, using the sandwich ELISA method of Erhardt et al. ${ }^{(19)}$. The laboratory participates in the US Centers for Disease Control and Prevention (CDC) VITAL-EQA inter-laboratory comparison rounds and has a rigorous internal quality control system.

The Swiss Vitamin Institute analysed plasma samples from WRA for folate concentrations using the microbiological assay and Lactobacillus caseii ATCC $7469^{(20)}$. On a sub-sample (random selection of half of the samples), vitamin $\mathrm{B}_{12}$ was analysed using the same method but working with Lactobacillus leichmanii (ATCC 7830). The laboratory regularly participates in the CDC interlaboratory comparison rounds.

\section{Data management and analysis}

All field data were doubly entered using a pre-programmed data-entry screen (Microsoft ${ }^{\circledR}$ Access, version 2000), merged and cross-checked. Laboratory data were either auto-generated or doubly entered (Microsoft ${ }^{\circledR}$ Excel, version 97-2003).

\section{Socio-economic classification}

The poverty index was based on household characteristics and assets including: quality of housing (type of roof, wall); access to electricity and water; possession of electronic equipment; and transport. Analysis was done based on a principal component analysis-based asset index ${ }^{(21)}$, using a method and formula by Gwatkin et al. ${ }^{(22)}$, and missing data were replaced prior to analysis with the mean of the asset. The discontinuous poverty index variable was then divided along predefined quintiles to assign each household to a socio-economic quintile, where 1 is the poorest and 5 the wealthiest quintile.

\section{Anthropometry}

Using the WHO 2006 growth standards, each child's $Z$-score for height-for-age (HAZ), weight-for-age (WAZ) and weight-for-height (WHZ) was determined. Z-scores have an overall mean set to 0 and sD of 1 . A child who has
HAZ of $\leq 2 \cdot 00$ is classified as stunted, a child with WAZ of $\leq 2 \cdot 00$ is classified as underweight and a child with WHZ of $\leq 2 \cdot 00$ is classified as wasted.

For WRA, BMI was calculated as [weight $(\mathrm{kg})] /[$ height $(\mathrm{m})]^{2}$ and four BMI categories were generated: underweight, $\mathrm{BMI}<18 \cdot 5 \mathrm{~kg} / \mathrm{m}^{2}$; normal, $\mathrm{BMI}=18 \cdot 5-24 \cdot 9 \mathrm{~kg} / \mathrm{m}^{2}$; overweight, $\quad$ BMI $=25 \cdot 0-29 \cdot 9 \mathrm{~kg} / \mathrm{m}^{2} ;$ and obese, BMI $\geq 30 \cdot 0 \mathrm{~kg} / \mathrm{m}^{2(23)}$. Both $Z$-scores and BMI were analysed as continuous and categorical variables.

\section{Blood parameters}

Anaemia is defined as $\mathrm{Hb}$ concentration of $<110 \mathrm{~g} / \mathrm{l}$ in children aged 6-59 months and $\mathrm{Hb}$ concentration of $<120 \mathrm{~g} / 1$ in non-pregnant WRA. At the population level, a $\geq 40 \%$ prevalence of $\mathrm{Hb}$ concentrations below the cut-off indicates a severe public health problem, prevalence of $20 \cdot 0-39 \cdot 9 \%$ indicates a moderate public health problem and prevalence of $5 \cdot 0-19 \cdot 9 \%$ a mild public health problem; where the prevalence is $<4.9 \%$, the population is described as normal ${ }^{(8)}$. Plasma sTfR concentrations reflect the intensity of erythropoiesis and the demand for Fe. The sTfR concentration rises in Fe-deficiency anaemia and is a marker of the severity of Fe insufficiency, but only when Fe stores have been exhausted and provided there are no other causes of abnormal erythropoiesis; concentrations of $>8.3 \mathrm{mg} / 1$ are considered abnormal ${ }^{(19)}$ and are classified as Fe-deficient erythropoiesis. Fedeficiency anaemia is defined as those with anaemia and Fe deficiency (low ferritin, as defined below). Low plasma folate status is indicated by folate concentrations $<10 \mathrm{nmol} / \mathrm{l}$ and vitamin $\mathrm{B}_{12}$ deficiency by vitamin $\mathrm{B}_{12}$ concentrations $<150 \mathrm{pmol} / \mathrm{l}^{(24)}$.

Ferritin concentrations of $<12 \mu \mathrm{g} / 1$ in children aged $<5$ years, of $<15 \mu \mathrm{g} / 1$ in all people aged $>5$ years in the absence of infection and of $<30 \mu \mathrm{g} / 1$ in the presence of infection indicate Fe deficiency ${ }^{(8)}$. Ferritin concentrations are increased by inflammation and hence the prevalence of Fe deficiency in populations is generally underestimated. Therefore, to interpret ferritin in the presence of inflammation, the WHO suggests that one or more acute-phase proteins should be measured to detect the presence of inflammation but no instruction on how to use acutephase proteins to interpret ferritin is provided ${ }^{(25)}$. Thurnham and colleagues ${ }^{(26)}$ described a method to estimate the increase in ferritin caused by inflammation in apparently healthy people using two acute-phase proteins, CRP and AGP, and calculated factors to adjust ferritin for the influence of inflammation ${ }^{(12)}$ : (i) no elevated acute-phase proteins, no correction; (ii) CRP concentration of $>5 \mathrm{mg} / \mathrm{l}$, correction factor of $0 \cdot 77$; (iii) CRP concentration $>5 \mathrm{mg} / \mathrm{l}$ and AGP concentration $>1 \mathrm{~g} / 1$, correction factor of $0 \cdot 53$; and (iv) AGP concentration $>1 \mathrm{~g} / \mathrm{l}$, correction factor of $0 \cdot 75$.

$\mathrm{VAD}$ is defined as a serum retinol concentration $<0.7 \mu \mathrm{mol} / \mathrm{l}^{(27)}$ and in populations a prevalence of $\geq 2-<10 \%$ indicates a mild, prevalence of $\geq 10-<20 \%$ a moderate and prevalence of $\geq 20 \%$ indicates a severe 
public health problem ${ }^{(28)}$. Retinol concentrations are reduced by the presence of inflammation and hence prevalence of VAD is overestimated. A similar correction to that for ferritin can also be done for retinol concentrations $^{(12)}$. In the present study, RBP concentrations were measured, and not retinol; but because there was an excellent correlation between the retinol and RBP concentrations in the CDC VITAL-EQA programme, the same thresholds were used. The correction for inflammation proposed for serum retinol was applied to adjust RBP concentrations: (i) no inflammation, no correction; (ii) elevated CRP, correction factor of $0 \cdot 13$; (iii) elevated CRP and AGP, correction factor of $0 \cdot 24$; and (iv) elevated AGP, correction factor of $0 \cdot 11$.

\section{Statistical analysis}

Data management and analysis were performed with the statistical software package IBM SPSS Statistics 19. All continuous data were checked for skewness using the Cox test (coefficient of skewness divided by the standard error of skewness) as well as by examination of the frequency distribution with a normal curve. All analyses took into account characteristics of the cluster sampling design. Dichotomous variables are expressed as percentages. Continuous variables are expressed as arithmetic means and standard deviations, except for non-normally distributed data that were log-transformed before statistical analysis and expressed as geometric means. Associations between prevalence and residency (urban/rural) were assessed using univariate logistic regression models. Associations between continuous variables and region or residency were assessed using univariate linear regression models. The first type error rate was set at $0 \cdot 05$. Wherever it is stated that the comparison yields a difference, this refers to a significant difference.

Logistic regression models with micronutrient prevalence as the response variable were used to assess the effect of the different factors (urban or rural area, socioeconomic class and age class for children).

\section{Results}

The questionnaire reported data from 990 households of which 447 were in the rural area. Apart from ownership of a motorcycle, there were urban/rural differences among all the other demographic categories (Table 1).

\section{Women of reproductive age}

Information was available on 928 non-pregnant WRA whose mean age was $27 \cdot 7$ ( $\mathrm{SD} 8 \cdot 0$ ) years. There were no differences in mean age by residency (urban/rural; Table 2). Weight, BMI, BMI categories and use of a bed net, but not height, were different by residency among the WRA.

Prevalence of malaria parasitaemia $(5 \cdot 1 \%)$ was low but there were rural/urban differences $(P<0 \cdot 007$; Table 3$)$. Inflammation, as indicated by the elevation of the acutephase proteins CRP and AGP, was found in $33.7 \%$ of the women, but there were no differences in exposure to inflammation according to residency.

The prevalence of anaemia was $49.9 \%$ and there were differences in prevalence of anaemia by residency, with

Table 1 Household characteristics of the study sample by residency (rural/urban), Côte d'Ivoire, 2007

\begin{tabular}{|c|c|c|c|c|c|}
\hline Household characteristics & Category (\%) & Rural (n 447) & Urban (n 543) & Total (n 990) & $P$ (rural $v$ urban) \\
\hline \multirow[t]{4}{*}{ Wall construction } & Mud & $23 \cdot 7$ & $0 \cdot 4$ & $10 \cdot 9$ & $<0.001$ \\
\hline & Wood & $4 \cdot 3$ & $4 \cdot 1$ & $4 \cdot 1$ & \\
\hline & Mud + cement & $37 \cdot 6$ & $5 \cdot 9$ & $20 \cdot 2$ & \\
\hline & Cement only & $34 \cdot 5$ & $89 \cdot 5$ & $64 \cdot 7$ & \\
\hline \multirow[t]{2}{*}{ Roof construction } & Paper/straw & $33 \cdot 0$ & $0 \cdot 2$ & $14 \cdot 9$ & $<0.001$ \\
\hline & Corrugated iron & $67 \cdot 0$ & $99 \cdot 8$ & $85 \cdot 1$ & \\
\hline \multirow[t]{4}{*}{ Cooking } & Wood & $87 \cdot 1$ & $25 \cdot 5$ & $53 \cdot 4$ & $<0.001$ \\
\hline & Charcoal & $11 \cdot 3$ & $43 \cdot 0$ & $28 \cdot 6$ & \\
\hline & Gas & $1 \cdot 6$ & $31 \cdot 0$ & $17 \cdot 7$ & \\
\hline & Electricity & - & 0.6 & 0.3 & \\
\hline Electricity & Yes & $31 \cdot 7$ & $94 \cdot 3$ & $34 \cdot 0$ & $<0.001$ \\
\hline Fridge & Yes & $4 \cdot 1$ & $25 \cdot 6$ & $16 \cdot 7$ & $<0.001$ \\
\hline Television & Yes & $29 \cdot 2$ & $73 \cdot 2$ & $55 \cdot 1$ & $<0.001$ \\
\hline Radio & Yes & $53 \cdot 8$ & $74 \cdot 1$ & $65 \cdot 8$ & $<0.001$ \\
\hline Fan & Yes & $14 \cdot 2$ & $67 \cdot 2$ & $45 \cdot 4$ & $<0.001$ \\
\hline Car & Yes & $0 \cdot 4$ & $5 \cdot 2$ & $3 \cdot 0$ & $<0.001$ \\
\hline Motorcycle & Yes & $13 \cdot 6$ & $13 \cdot 4$ & $13 \cdot 5$ & NS \\
\hline Bicycle & Yes & $47 \cdot 3$ & $12 \cdot 1$ & $28 \cdot 0$ & $<0.001$ \\
\hline \multirow[t]{4}{*}{ Toilet } & Open & $55 \cdot 7$ & $11 \cdot 6$ & $31 \cdot 4$ & $<0.001$ \\
\hline & Pit latrine & $13 \cdot 3$ & $2 \cdot 6$ & $7 \cdot 4$ & \\
\hline & Cement latrine & $28 \cdot 5$ & $63 \cdot 6$ & $47 \cdot 8$ & \\
\hline & Flush toilet & $2 \cdot 5$ & $22 \cdot 2$ & $13 \cdot 3$ & \\
\hline \multirow[t]{5}{*}{ Water source } & River & $2 \cdot 3$ & - & $1 \cdot 0$ & $<0.001$ \\
\hline & Central water pump & $58 \cdot 8$ & $8 \cdot 0$ & $31 \cdot 0$ & \\
\hline & Private tubewell & $16 \cdot 0$ & $15 \cdot 4$ & $15 \cdot 7$ & \\
\hline & Public tubewell & $20 \cdot 3$ & $2 \cdot 0$ & $10 \cdot 3$ & \\
\hline & Tap water & $2 \cdot 7$ & $74 \cdot 5$ & $42 \cdot 1$ & \\
\hline
\end{tabular}


Table 2 Age, anthropometry and bed-net use in the study sample by residency (rural/urban): women of reproductive age (aged 15-49 years) and pre-school age children (aged 6-59 months), Côte d'Ivoire, 2007

\begin{tabular}{|c|c|c|c|c|c|c|c|}
\hline \multirow[b]{2}{*}{ Women of reproductive age } & \multicolumn{2}{|c|}{ Rural } & \multicolumn{2}{|c|}{ Urban } & \multicolumn{2}{|c|}{ Total } & \multirow[b]{2}{*}{$P$ (rural $v$ urban) } \\
\hline & Mean & SD & Mean & SD & Mean & SD & \\
\hline Age (years) & $27 \cdot 8$ & $7 \cdot 6$ & $27 \cdot 5$ & $8 \cdot 0$ & $27 \cdot 7$ & $8 \cdot 0$ & NS \\
\hline Height (cm) & $159 \cdot 2$ & $6 \cdot 0$ & $159 \cdot 7$ & $6 \cdot 2$ & $159 \cdot 5$ & $6 \cdot 1$ & NS \\
\hline Weight (kg) & $55 \cdot 8$ & $9 \cdot 5$ & $60 \cdot 7$ & $12 \cdot 9$ & $58 \cdot 5$ & $11 \cdot 7$ & $<0.001$ \\
\hline \multirow[t]{2}{*}{ BMI $\left(\mathrm{kg} / \mathrm{m}^{2}\right)$} & $22 \cdot 0$ & $3 \cdot 6$ & $23 \cdot 7$ & $4 \cdot 7$ & $23 \cdot 0$ & $4 \cdot 4$ & $<0.001$ \\
\hline & \multicolumn{2}{|c|}{$\%$} & \multicolumn{2}{|c|}{$\%$} & \multicolumn{2}{|c|}{$\%$} & \\
\hline \multicolumn{8}{|l|}{ BMI category $\left(\mathrm{kg} / \mathrm{m}^{2}\right)$} \\
\hline$<18.5$ & \multicolumn{2}{|c|}{$11 \cdot 5$} & \multicolumn{2}{|c|}{$5 \cdot 9$} & \multicolumn{2}{|c|}{$8 \cdot 4$} & $<0.001$ \\
\hline $18 \cdot 5-24 \cdot 9$ & \multicolumn{2}{|c|}{$73 \cdot 6$} & \multicolumn{2}{|c|}{$62 \cdot 9$} & \multicolumn{2}{|c|}{$67 \cdot 8$} & \\
\hline $25 \cdot 0-29 \cdot 9$ & \multirow{2}{*}{\multicolumn{2}{|c|}{$\begin{array}{r}10 \cdot 4 \\
4 \cdot 5\end{array}$}} & \multirow{2}{*}{\multicolumn{2}{|c|}{$\begin{array}{r}22 \cdot 0 \\
9 \cdot 2\end{array}$}} & \multicolumn{2}{|c|}{$\begin{array}{r}16 \cdot 7 \\
7 \cdot 1\end{array}$} & \\
\hline$\geq 30 \cdot 0$ & & & & & & & \\
\hline \multirow[t]{2}{*}{ Sleeping under a bed net (\%) } & \multicolumn{2}{|c|}{$28 \cdot 9$} & \multicolumn{2}{|c|}{$17 \cdot 0$} & \multicolumn{2}{|c|}{$22 \cdot 5$} & $<0 \cdot 001$ \\
\hline & \multicolumn{2}{|c|}{ Rural } & \multicolumn{2}{|c|}{ Urban } & \multicolumn{2}{|c|}{ Total } & \\
\hline Pre-school age children & Mean & SD & Mean & SD & Mean & SD & $P$ (rural $v$ urban) \\
\hline Age (months) & $30 \cdot 4$ & $15 \cdot 2$ & $30 \cdot 7$ & $15 \cdot 1$ & $30 \cdot 6$ & $15 \cdot 1$ & NS \\
\hline Height $(\mathrm{cm})$ & $83 \cdot 8$ & $13 \cdot 0$ & 85.9 & $13 \cdot 7$ & 84.9 & $13 \cdot 4$ & 0.023 \\
\hline Weight (kg) & $11 \cdot 1$ & $3 \cdot 8$ & $11 \cdot 8$ & 3.5 & $11 \cdot 5$ & $3 \cdot 7$ & 0.002 \\
\hline HAZ & $-1 \cdot 74$ & $1 \cdot 83$ & $-1 \cdot 30$ & $1 \cdot 57$ & $-1 \cdot 51$ & $1 \cdot 71$ & $<0.001$ \\
\hline WAZ & $-1 \cdot 44$ & 1.59 & -0.93 & $1 \cdot 41$ & $-1 \cdot 17$ & 1.52 & $<0.001$ \\
\hline \multirow[t]{2}{*}{ WHZ } & -0.56 & $1 \cdot 50$ & -0.31 & $1 \cdot 55$ & $-0 \cdot 43$ & $1 \cdot 53$ & $<0.001$ \\
\hline & \multicolumn{2}{|c|}{$\%$} & & & & & \\
\hline Stunting & & & & & & & \\
\hline Normal & & & & & & & $<0.001$ \\
\hline Stunted $(\mathrm{HAZ}=-2 \cdot 00$ to -2.99$)$ & & & & & & & \\
\hline Severely stunted $(\mathrm{HAZ}<-3 \cdot 00)$ & & & & & & & \\
\hline Underweight & & & & & & & \\
\hline Normal & & & & & & & $<0.001$ \\
\hline Underweight (WAZ $=-2.00$ to -2.99$)$ & & & & & & & \\
\hline Severely underweight $(\mathrm{WAZ}<-3.00)$ & & & & & & & \\
\hline Wasting & & & & & & & \\
\hline Normal & & & & & & & NS \\
\hline Wasted $(\mathrm{WHZ}=-2.00$ to -2.99$)$ & & & & & & & \\
\hline Severely wasted $(\mathrm{WHZ}<-3.00)$ & & & & & & & \\
\hline Sleeping under a bed net (\%) & & & & & & & $<0.001$ \\
\hline
\end{tabular}

HAZ, height-for-age Z-score; WAZ, weight-for-age Z-score; WHZ, weight-for-height Z-score.

more anaemia in rural $(55 \cdot 1 \%)$ compared with urban (45.6\%) areas $(P=0.004$; Table 3$)$. Prevalence of anaemia in WRA was different by eco-region with those in the south having the highest prevalence $(63 \cdot 1 \%, P=0 \cdot 001$; Table 4).

There were differences in both the uncorrected and corrected ferritin concentrations by residency and the prevalence of Fe deficiency, using the corrected data, was higher in the urban areas (19.6\%) compared with the rural areas $(13.5 \%, P=0 \cdot 014$; Table 3$)$. Fe deficiency and Fe-deficiency anaemia were not different by eco-region (Table 4).

The mean RBP concentration was higher in the rural (1.60 $\mu \mathrm{mol} / \mathrm{l})$ compared with the urban $(1.51 \mu \mathrm{mol} / \mathrm{l})$ areas $(P=0 \cdot 004 ;$ Table 3$)$. There were no differences in VAD by eco-region (Table 4 ).

Mean folate concentrations were less than the defined cut-off of $10 \mathrm{nmol} / \mathrm{l}^{(24)}$ and both folate levels and the prevalence of folate deficiency were worse (both $P<0 \cdot 001$ ) in the urban $(4.6 \mathrm{nmol} / 1$ and $91.2 \%$, respectively) than rural areas $(7 \cdot 4 \mathrm{nmol} / 1$ and $80 \cdot 4 \%$, respectively; Table 3$)$.
The prevalence of folate deficiency varied by eco-region, with populations in the north-west and Abidjan having the highest prevalence $(\sim 96 \%, P<0 \cdot 001)$. Folate concentrations were not significantly associated with $\mathrm{Hb}$ concentrations (data not shown).

Mean concentrations of vitamin $\mathrm{B}_{12}$ were in the normal range $(>150 \mathrm{pmol} / \mathrm{l})$, but the prevalence of those with vitamin $\mathrm{B}_{12}$ deficiency was higher in rural (24.2\%) compared with urban $(12 \cdot 8 \%)$ areas $(P=0 \cdot 002$; Table 3$)$. The prevalence of vitamin $\mathrm{B}_{12}$ deficiency was markedly different by eco-region $(P<0 \cdot 001)$, especially between the north $(48 \cdot 6 \%)$ and south $(6 \cdot 2 \%$; Table 4$)$.

\section{Pre-school age children}

Data were available from 879 pre-SAC, of whom $47 \cdot 3 \%$ were female (sex ratio, male:female $=1 \cdot 08: 1$; Table 5 ). The mean age of the pre-SAC was $30 \cdot 6$ (SD 15.1) months and there were no differences in mean age by residency (urban/rural; Table 2). The prevalences of stunting $(46 \cdot 2 \%)$, wasting $(14 \cdot 1 \%)$ and underweight $(29 \cdot 0 \%)$ 
Table 3 Vitamin and mineral status and prevalence of deficiencies by residency (rural/urban): women of reproductive age (aged 15-49 years), Côte d'Ivoire, 2007

\begin{tabular}{|c|c|c|c|c|c|c|c|c|c|c|}
\hline & \multicolumn{3}{|c|}{ Rural } & \multicolumn{3}{|c|}{ Urban } & \multicolumn{3}{|c|}{ Total } & \multirow[b]{2}{*}{$P$ (rural $v$ urban) } \\
\hline & $n$ & Mean or prevalence & SD & $n$ & Mean or prevalence & SD & $n$ & Mean or prevalence & SD & \\
\hline \multicolumn{11}{|l|}{ Plasmodium spp. } \\
\hline Intensity (parasites $/ \mu$ l blood) ${ }^{\star}$ & 30 & $504 \cdot 1$ & $1113 \cdot 5$ & 15 & $578 \cdot 8$ & $566 \cdot 0$ & 45 & $529 \cdot 2$ & $957 \cdot 7$ & NS \\
\hline Parasitaemia (\%) & 415 & $7 \cdot 2$ & - & 491 & $3 \cdot 3$ & - & 906 & $5 \cdot 1$ & - & 0.007 \\
\hline \multicolumn{11}{|l|}{ Fe biomarkers } \\
\hline $\mathrm{Hb}(\mathrm{g} / \mathrm{l})$ & 425 & $117 \cdot 4$ & $18 \cdot 3$ & 503 & $119 \cdot 3$ & $15 \cdot 8$ & 928 & $118 \cdot 4$ & $17 \cdot 0$ & NS \\
\hline Anaemia (\%)† & 234 & $55 \cdot 1$ & - & 229 & $45 \cdot 6$ & - & 463 & $49 \cdot 9$ & - & 0.004 \\
\hline Ferritin $(\mu \mathrm{g} / \mathrm{l}) \ddagger$ & 423 & $65 \cdot 8$ & $79 \cdot 8$ & 483 & $55 \cdot 7$ & $59 \cdot 9$ & 906 & $60 \cdot 4$ & $70 \cdot 0$ & 0.030 \\
\hline Ferritin, corrected $(\mu \mathrm{g} / \mathrm{l}) \ddagger$ & 423 & $56 \cdot 4$ & $63 \cdot 5$ & 483 & $47 \cdot 1$ & $46 \cdot 7$ & 906 & $51 \cdot 5$ & $55 \cdot 3$ & 0.011 \\
\hline $\mathrm{sTfR}(\mathrm{mg} / \mathrm{l})$ & 422 & $5 \cdot 4$ & $2 \cdot 6$ & 485 & 4.9 & $2 \cdot 1$ & 907 & $5 \cdot 1$ & 4.5 & $<0.001$ \\
\hline Fe deficiency (\%)† & 57 & $13 \cdot 5$ & - & 95 & $19 \cdot 6$ & - & 152 & $16 \cdot 7$ & - & 0.014 \\
\hline Fe-deficient erythropoiesis (\%)t,§ & 24 & $5 \cdot 7$ & - & 37 & $7 \cdot 6$ & - & 61 & $6 \cdot 7$ & - & NS \\
\hline Fe-deficiency anaemia (\%)t,§ & 42 & $9 \cdot 9$ & - & 63 & $13 \cdot 3$ & - & 105 & $11 \cdot 6$ & - & NS \\
\hline \multicolumn{11}{|l|}{ Vitamin A status } \\
\hline $\mathrm{RBP}(\mu \mathrm{mol} / \mathrm{l})$ & 422 & $1 \cdot 60$ & 0.52 & 485 & $1 \cdot 51$ & 0.43 & 907 & 1.55 & 0.48 & 0.004 \\
\hline VAD (\%)t & 3 & $0 \cdot 7$ & - & 4 & $0 \cdot 8$ & - & 7 & $0 \cdot 8$ & - & NS \\
\hline \multicolumn{11}{|l|}{ Folate and vitamin $B_{12}$ status } \\
\hline Plasma folate $(\mathrm{nmol} / \mathrm{l})$ & 407 & $7 \cdot 4$ & $9 \cdot 5$ & 446 & $4 \cdot 6$ & $5 \cdot 6$ & 853 & $5 \cdot 9$ & $7 \cdot 9$ & $<0.001$ \\
\hline Folate deficiency $(\%) \dagger$ & 327 & $80 \cdot 4$ & - & 407 & $91 \cdot 2$ & - & 734 & $86 \cdot 1$ & - & $<0.001$ \\
\hline Plasma vitamin $B_{12}(\mathrm{pmol} / \mathrm{l}) \|$ & 211 & $307 \cdot 2$ & $210 \cdot 1$ & 243 & $364 \cdot 9$ & $201 \cdot 3$ & 454 & $338 \cdot 1$ & $207 \cdot 2$ & 0.003 \\
\hline Vitamin $\mathrm{B}_{12}$ deficiency $(\%)+, \|$ & 51 & $24 \cdot 2$ & - & 31 & $12 \cdot 8$ & - & 82 & $18 \cdot 1$ & - & 0.002 \\
\hline \multicolumn{11}{|l|}{ Inflammation } \\
\hline CRP (mg/l) & 422 & $3 \cdot 7$ & $6 \cdot 3$ & 485 & $4 \cdot 0$ & $6 \cdot 1$ & 907 & $3 \cdot 8$ & $6 \cdot 2$ & NS \\
\hline AGP $(\mathrm{g} / \mathrm{l})$ & 422 & 0.90 & $0 \cdot 3$ & 485 & $0 \cdot 84$ & $0 \cdot 3$ & 907 & $0 \cdot 86$ & $0 \cdot 3$ & 0.04 \\
\hline \multicolumn{11}{|l|}{ Inflammation (\%) } \\
\hline None & 281 & $66 \cdot 6$ & - & 320 & $66 \cdot 0$ & - & 601 & $66 \cdot 3$ & - & NS \\
\hline Elevated CRP & 27 & $6 \cdot 4$ & - & 47 & $9 \cdot 7$ & - & 74 & $8 \cdot 1$ & - & \\
\hline Elevated CRP and AGP & 47 & $11 \cdot 1$ & - & 63 & $13 \cdot 0$ & - & 110 & $12 \cdot 1$ & - & \\
\hline Elevated AGP & 67 & $15 \cdot 9$ & - & 55 & $11 \cdot 3$ & - & 122 & $13 \cdot 4$ & - & \\
\hline
\end{tabular}

STfR; soluble transferrin receptor; RBP, retinol-binding protein; VAD, vitamin A deficiency; CRP, C-reactive protein; AGP, $\alpha_{1}$-acid-glycoprotein.

*Geometric mean.

tAnaemia, $\mathrm{Hb}<120 \mathrm{~g} / \mathrm{l}$; Fe deficiency, ferritin $<15 \mu \mathrm{g} /$; Fe-deficient erythropoiesis, sTfR $>8.3 \mathrm{mg} / \mathrm{l}$; Fe-deficiency anaemia, ferritin $<15 \mu \mathrm{g} / \mathrm{l}$ and $\mathrm{Hb}<120 \mathrm{~g} / \mathrm{l}$; VAD, RBP $<0.7 \mu \mathrm{mol} / /$; folate deficiency, plasma folate $<10 \mathrm{nmol} / /$; vitamin $\mathrm{B}_{12}$ deficiency, plasma vitamin $\mathrm{B}_{12}<150 \mathrm{pmol} / \mathrm{l}$.

¥Median.

$\S$ Using corrected ferritin values.

IIRandom sub-sample of half of women of reproductive age. 
Table 4 Prevalence of deficiencies of iron, vitamin A, folate and vitamin $B_{12}$ by eco-region: women of reproductive age (aged 15-49 years), Côte d'Ivoire, 2007

\begin{tabular}{|c|c|c|c|c|c|c|c|}
\hline Eco-region & Anaemia* (\%) & $\begin{array}{c}\mathrm{Fe} \\
\text { deficiencyt (\%) }\end{array}$ & $\begin{array}{c}\text { Fe-deficient } \\
\text { erythropoiesis (\%) }\end{array}$ & $\begin{array}{l}\text { Fe-deficiency } \\
\text { anaemia (\%) }\end{array}$ & VAD (\%) & $\begin{array}{c}\text { Folate } \\
\text { deficiency (\%) }\end{array}$ & $\begin{array}{c}\text { Vitamin } \mathrm{B}_{12} \\
\text { deficiency } ¥ \text { (\%) }\end{array}$ \\
\hline West & $52 \cdot 8$ & $22 \cdot 1$ & $9 \cdot 6$ & $10 \cdot 6$ & - & $77 \cdot 5$ & $29 \cdot 8$ \\
\hline North-west & $37 \cdot 9$ & $28 \cdot 6$ & $7 \cdot 1$ & $17 \cdot 9$ & - & $96 \cdot 4$ & $36 \cdot 4$ \\
\hline North & $54 \cdot 7$ & $15 \cdot 6$ & $7 \cdot 8$ & $12 \cdot 7$ & $1 \cdot 6$ & $88 \cdot 3$ & $48 \cdot 6$ \\
\hline North-east & $44 \cdot 0$ & $15 \cdot 4$ & $6 \cdot 7$ & 8.9 & - & $78 \cdot 7$ & $40 \cdot 4$ \\
\hline Central & $38 \cdot 0$ & $14 \cdot 1$ & $7 \cdot 0$ & $7 \cdot 1$ & - & $74 \cdot 2$ & $20 \cdot 0$ \\
\hline Central-west & $50 \cdot 0$ & $21 \cdot 0$ & $7 \cdot 0$ & $16 \cdot 8$ & $3 \cdot 0$ & $83 \cdot 7$ & $11 \cdot 5$ \\
\hline South-east & $54 \cdot 3$ & $15 \cdot 4$ & $4 \cdot 8$ & $11 \cdot 7$ & - & $92 \cdot 1$ & $7 \cdot 0$ \\
\hline South & $63 \cdot 1$ & $13 \cdot 6$ & $7 \cdot 7$ & $12 \cdot 5$ & $1 \cdot 2$ & $89 \cdot 8$ & $6 \cdot 2$ \\
\hline Abidjan & $42 \cdot 5$ & $15 \cdot 4$ & $4 \cdot 7$ & $10 \cdot 1$ & $0 \cdot 7$ & $96 \cdot 2$ & - \\
\hline$P$ & 0.001 & NS & NS & NS & NS & $<0.001$ & $<0.001$ \\
\hline
\end{tabular}

VAD, vitamin A deficiency; sTfR; soluble transferrin receptor; RBP, retinol-binding protein.

${ }^{*}$ Anaemia, $\mathrm{Hb}<120 \mathrm{~g} / \mathrm{l}$; Fe deficiency, ferritin $<15 \mu \mathrm{g} / \mathrm{l}$; Fe-deficient erythropoiesis, sTFR $>8.3 \mathrm{mg} / \mathrm{l} ;$ Fe-deficiency anaemia, ferritin $<15 \mu \mathrm{g} / \mathrm{l}$ and $\mathrm{Hb}<120 \mathrm{~g} / \mathrm{l}$; $\mathrm{VAD}, \mathrm{RBP}<0.7 \mu \mathrm{mol} / \mathrm{l}$; folate deficiency, plasma folate $<10 \mathrm{nmol} / \mathrm{l}$; vitamin $\mathrm{B}_{12}$ deficiency, plasma vitamin $\mathrm{B}_{12}<150 \mathrm{pmol} / \mathrm{l}$.

†Adjusted for inflammation.

‡Random sub-sample of half of women of reproductive age.

were high (Table 2). Use of bed nets and all anthropometric measures, apart from categories of wasting, were different by residency $(P<0 \cdot 001$; Table 2$)$.

Children had high loads of Plasmodium spp. parasites (Table 5) and the prevalence of parasitaemia was higher in the rural $(38.7 \%)$ than the urban areas $(14.5 \%$, $P=0.001)$; however, when age group and residency were combined, there were no differences in prevalence (Fig. 1). Mean CRP and AGP concentrations were higher than the defined cut-offs, indicating the presence of inflammation, with more inflammation in rural $(78.5 \%)$ than urban $(56.9 \%)$ children $(P<0 \cdot 001$; Table 5$)$.

Mean $\mathrm{Hb}$ concentrations were $<110 \mathrm{~g} / 1$ and children in the rural areas had lower mean concentrations $(95.9 \mathrm{~g} / \mathrm{l})$ than those in the urban areas $(101.5 \mathrm{~g} / 1, P<0 \cdot 001$; Table 5). Prevalence of anaemia was also higher in the rural (76.0\%) compared with the urban $(68.2 \%)$ areas $(P=0.009$; Table 5). In both urban and rural areas and in all eco-regions, anaemia was classified as a severe public health problem ${ }^{(8)}$. Prevalence of anaemia was different $(P<0 \cdot 001)$ by age group in urban but not rural children (Fig. 1). Anaemia was also different by eco-region, as those in the north had the highest prevalence $(86.4 \%$; Table 6) compared with the west where the prevalence was lowest $(59 \cdot 6 \% ; P<0.001)$.

The geometric mean of the ferritin concentrations, whether corrected or uncorrected, was $>12 \mu \mathrm{g} / \mathrm{l}^{(8)}$ and those in rural areas had significantly higher concentrations $(60.5 \mu \mathrm{g} / \mathrm{l})$ than those in urban areas $(42.9 \mu \mathrm{g} / \mathrm{l}$, $P<0 \cdot 001$; Table 5). Additionally, children with inflammation and Plasmodium parasites had mean adjusted ferritin concentrations that were $12 \mu \mathrm{g} / 1$ higher than in children without Plasmodium parasites. Fe deficiency was different by age group in both the rural $(P=0 \cdot 005)$ and urban areas $(P<0 \cdot 001$; Fig. 1). Prevalence of Fe-deficiency anaemia was considerably higher in the urban (17.5\%) than rural $(6 \cdot 3 \%)$ areas $(P<0 \cdot 001$; Table 5$)$ and there was a big variation by eco-region, from $3.6 \%$ in the west to $27 \cdot 8 \%$ in the northwest $(P<0 \cdot 001$; Table 6$)$.
Children with Fe deficiency had sTfR concentrations that were higher ( $n$ 87, mean $13.8 \mathrm{mg} / \mathrm{l}$ ) than those without ( $n 695$, mean $10.3 \mathrm{mg} / 1 ; P<0 \cdot 001$ ). Children with anaemia and Plasmodium parasites had higher STfR concentrations $(14.3 \mathrm{mg} / \mathrm{l})$ than children without $(10.4 \mathrm{mg} / 1 ; \quad P<0 \cdot 001)$. Fe-deficient erythropoiesis was higher in the urban than rural areas $(P<0 \cdot 001$; Table 5) but did not differ among eco-regions (Table 6 ).

The prevalence of RBP concentrations $<0.7 \mu \mathrm{mol} / 1$, the population-specific threshold, was $24 \cdot 1 \%$, which according to the WHO criteria makes VAD a severe public health problem in this age group. There were differences in RBP concentrations between urban $(0.99 \mu \mathrm{mol} / \mathrm{l})$ and rural $(0.87 \mu \mathrm{mol} / \mathrm{l})$ populations $(P=0.012$; Table 5$)$ and there were differences in VAD by age group in rural areas ( $P=0 \cdot 014$; Fig. 1$)$. VAD also differed by eco-region, with children in the south-east having the best status $(12 \cdot 4 \%$, $P<0 \cdot 001$; Table 6).

Further examination of the biochemical and anthropometric data by the poverty index quintiles, age, sex and residency using multiple regression analysis found an association between increase in age and $\mathrm{Hb}$ concentration of $+0 \cdot 294 \mathrm{~g} / 1$ per month. There was also an association between wealth quintile and $\mathrm{Hb}$ concentration. Using quintile 5 as the reference group, those in the other quintiles had lower $\mathrm{Hb}$ concentrations: quintile $1,-16 \cdot 1 \mathrm{~g} / \mathrm{l}$ ( $n$ 176); quintile $2,-31 \cdot 5 \mathrm{~g} / \mathrm{l}$ ( $n$ 170); quintile $3,-12 \cdot 8 \mathrm{~g} / \mathrm{l}$ ( $n$ 161); and quintile $4,-12 \cdot 8 \mathrm{~g} / \mathrm{l}(n 161 ; P<0 \cdot 001)$. Finally, there was an association between quintile, residency and prevalence of Plasmodium spp. $(P<0 \cdot 001)$, with those pre-SAC in the poorest rural households having more Plasmodium infection than those in other quintiles (Fig. 2).

\section{Discussion}

In the present survey, demographic, nutritional and anthropometric data were collected from pre-SAC and 
Table 5 Vitamin and mineral status and prevalence of deficiencies by residency (rural/urban): pre-school age children (aged 6-59 months), Côte d'Ivoire, 2007

\begin{tabular}{|c|c|c|c|c|c|c|c|c|c|c|}
\hline & \multicolumn{3}{|c|}{ Rural } & \multicolumn{3}{|c|}{ Urban } & \multicolumn{3}{|c|}{ Total } & \multirow[b]{2}{*}{$P$ (rural $v$ urban) } \\
\hline & $n$ & Mean or prevalence & SD & $n$ & Mean or prevalence & SD & $n$ & Mean or prevalence & SD & \\
\hline Female (\%) & 411 & $46 \cdot 7$ & - & 468 & $47 \cdot 9$ & - & 879 & $47 \cdot 3$ & - & NS \\
\hline \multicolumn{11}{|l|}{ Plasmodium spp. } \\
\hline Intensity (parasites $/ \mu$ l blood) ${ }^{\star}$ & 152 & $3362 \cdot 4$ & $1133 \cdot 2$ & 66 & $6270 \cdot 9$ & $14416 \cdot 0$ & 218 & $4240 \cdot 2$ & $12384 \cdot 5$ & NS \\
\hline Parasitaemia (\%) & 393 & $38 \cdot 7$ & - & 455 & $14 \cdot 5$ & - & 848 & $25 \cdot 7$ & - & 0.001 \\
\hline \multicolumn{11}{|l|}{ Fe biomarkers } \\
\hline $\mathrm{Hb}(\mathrm{g} / \mathrm{l})$ & 411 & $95 \cdot 9$ & $19 \cdot 0$ & 468 & $101 \cdot 5$ & $17 \cdot 5$ & 879 & $98 \cdot 9$ & $18 \cdot 4$ & $<0.001$ \\
\hline Anaemia (\%)† & 411 & $76 \cdot 0$ & - & 468 & $68 \cdot 2$ & - & 879 & $71 \cdot 8$ & - & 0.009 \\
\hline Ferritin $(\mu \mathrm{g} / \mathrm{l}) \ddagger$ & 383 & $95 \cdot 6$ & $110 \cdot 3$ & 400 & $60 \cdot 7$ & $92 \cdot 0$ & 783 & $77 \cdot 7$ & $10 \cdot 3$ & $<0.001$ \\
\hline Ferritin, corrected $(\mu \mathrm{g} / \mathrm{l}) \ddagger$ & 383 & $60 \cdot 5$ & $63 \cdot 7$ & 400 & $42 \cdot 9$ & $56 \cdot 5$ & 783 & $51 \cdot 5$ & $60 \cdot 8$ & $<0.001$ \\
\hline $\operatorname{sTfR}(\mathrm{mg} / \mathrm{l})$ & 383 & $7 \cdot 4$ & $3 \cdot 1$ & 400 & $6 \cdot 7$ & $2 \cdot 8$ & 783 & $7 \cdot 0$ & $3 \cdot 0$ & 0.001 \\
\hline Fe deficiency (\%)† & 33 & $8 \cdot 6$ & - & 88 & $22 \cdot 1$ & - & 121 & $15 \cdot 5$ & - & $<0.001$ \\
\hline Fe-deficient erythropoiesis (\%)t,§ & 27 & $7 \cdot 0$ & - & 61 & $15 \cdot 3$ & - & 88 & $11 \cdot 2$ & - & $<0.001$ \\
\hline Fe-deficiency anaemia (\%)t,§ & 24 & $6 \cdot 3$ & - & 7 & $17 \cdot 5$ & - & 94 & $12 \cdot 0$ & - & $<0.001$ \\
\hline \multicolumn{11}{|l|}{ Vitamin A status } \\
\hline $\mathrm{RBP}(\mu \mathrm{mol} / \mathrm{l})$ & 383 & 0.87 & $0 \cdot 30$ & 399 & 0.99 & 0.33 & 782 & 0.93 & $0 \cdot 32$ & $<0.001$ \\
\hline VAD (\%)† & 107 & $28 \cdot 0$ & - & 81 & $20 \cdot 3$ & - & 188 & $24 \cdot 1$ & - & 0.012 \\
\hline \multicolumn{11}{|l|}{ Inflammation } \\
\hline CRP (mg/l) & 383 & $10 \cdot 4$ & $12 \cdot 3$ & 400 & $7 \cdot 1$ & $11 \cdot 4$ & 783 & $8 \cdot 7$ & $11 \cdot 9$ & $<0.001$ \\
\hline AGP $(g / l)$ & 383 & $1 \cdot 4$ & 0.45 & 400 & $1 \cdot 2$ & 0.46 & 783 & $1 \cdot 3$ & 0.47 & $<0.001$ \\
\hline \multicolumn{11}{|l|}{ Inflammation (\%) } \\
\hline None & 82 & $21 \cdot 5$ & - & 172 & $43 \cdot 1$ & - & 255 & $32 \cdot 6$ & - & $<0.001$ \\
\hline Elevated CRP & 13 & $3 \cdot 4$ & - & 10 & $2 \cdot 5$ & - & 23 & $2 \cdot 9$ & - & \\
\hline Elevated CRP and AGP & 180 & $47 \cdot 0$ & - & 113 & $28 \cdot 1$ & - & 292 & $37 \cdot 3$ & - & \\
\hline Elevated AGP & 108 & $28 \cdot 1$ & - & 105 & $26 \cdot 3$ & - & 213 & $27 \cdot 2$ & - & \\
\hline
\end{tabular}

STfR; soluble transferrin receptor; RBP, retinol-binding protein; VAD, vitamin A deficiency; CRP, C-reactive protein; AGP, $\alpha_{1}$-acid-glycoprotein

*Geometric mean.
+Anaemia, $\mathrm{Hb}<110 \mathrm{~g} /$; Fe deficiency, ferritin $<12 \mu \mathrm{g} /$; Fe-deficient erythropoiesis, sfTR $>8 \cdot 3 \mathrm{mg} /$; Fe-deficiency anaemia, ferritin $<12 \mu \mathrm{g} / \mathrm{l}$ and $\mathrm{Hb}<110 \mathrm{~g} /$; $\mathrm{VAD}, \mathrm{RBP}<0 \cdot 7 \mu \mathrm{mol} / \mathrm{l}$.

§Using corrected ferritin values. 


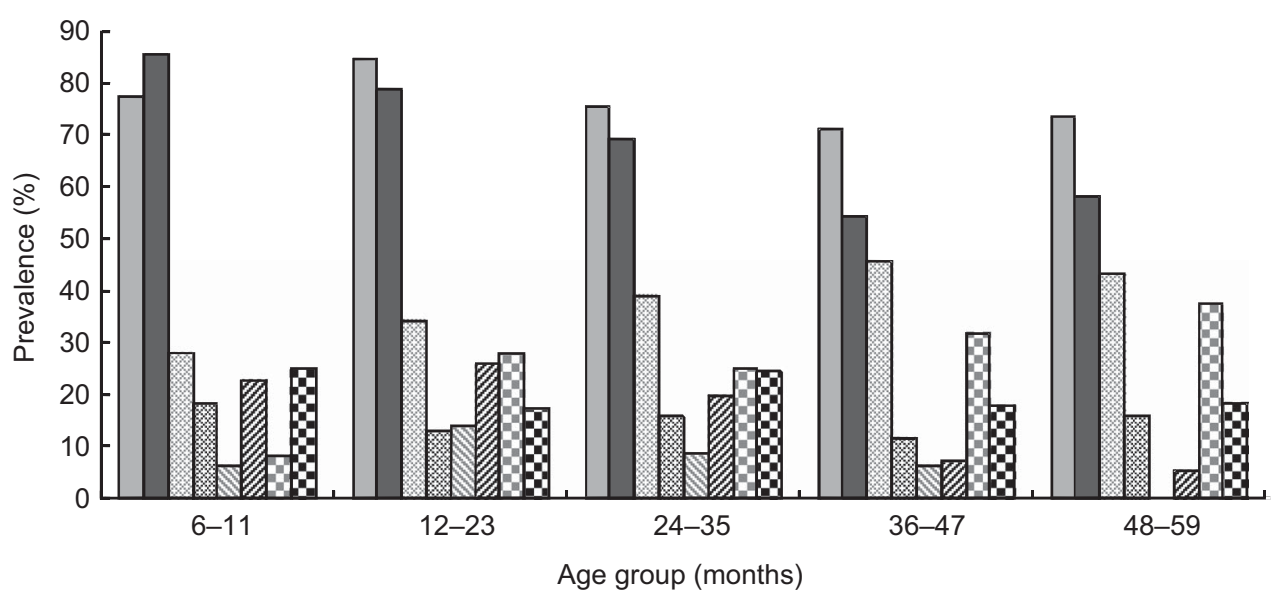

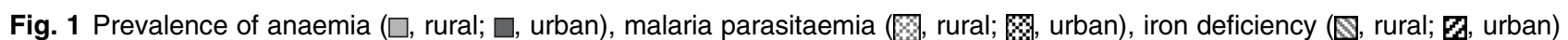
and vitamin A deficiency ( $\mathbf{B}$, rural; $\mathbf{\mathbf { H }}$, urban) by age and residency in pre-school children aged 6-59 months, Côte d'Ivoire, 2007. Anaemia, $\mathrm{Hb}<110 \mathrm{~g} / \mathrm{l}$; iron deficiency, ferritin $<12 \mu \mathrm{g} / \mathrm{l}$; vitamin A deficiency, retinol-binding protein $<0 \cdot 7 \mu \mathrm{mol} / \mathrm{l}$

Table 6 Prevalence of anaemia and deficiencies of iron and vitamin A by eco-region: pre-school age children (aged 6-59 months), Côte d'Ivoire, 2007

\begin{tabular}{|c|c|c|c|c|c|}
\hline Eco-region & Anaemia* $(\%)$ & Fe deficiency $(\%)$ & Fe-deficient erythropoiesis (\%) & Fe-deficiency anaemia (\%) & VAD (\%) \\
\hline West & $59 \cdot 6$ & $8 \cdot 4$ & $6 \cdot 0$ & $3 \cdot 6$ & $33 \cdot 3$ \\
\hline North-west & $66 \cdot 7$ & $44 \cdot 4$ & $16 \cdot 7$ & $27 \cdot 8$ & $33 \cdot 3$ \\
\hline North & $86 \cdot 4$ & $18 \cdot 4$ & $12 \cdot 2$ & $16 \cdot 3$ & $28 \cdot 6$ \\
\hline North-east & $68 \cdot 1$ & $16 \cdot 1$ & $14 \cdot 9$ & $13 \cdot 8$ & $23 \cdot 0$ \\
\hline Central & $79 \cdot 4$ & $16 \cdot 3$ & $10 \cdot 9$ & $14 \cdot 1$ & $13 \cdot 0$ \\
\hline Central-west & $81 \cdot 9$ & $19 \cdot 0$ & $13 \cdot 3$ & $16 \cdot 7$ & $33 \cdot 3$ \\
\hline South-east & $63 \cdot 1$ & $10 \cdot 5$ & $7 \cdot 6$ & $2 \cdot 9$ & $12 \cdot 4$ \\
\hline South & $84 \cdot 3$ & $8 \cdot 6$ & $7 \cdot 2$ & $8 \cdot 6$ & $31 \cdot 4$ \\
\hline Abidjan & $61 \cdot 2$ & $25 \cdot 7$ & $17 \cdot \overline{0}$ & $19 \cdot 8$ & $17 \cdot 9$ \\
\hline$P$ & $<0.001$ & $<0.001$ & NS & $<0.001$ & $<0.001$ \\
\hline
\end{tabular}

VAD, vitamin A deficiency; sTfR; soluble transferrin receptor; RBP, retinol-binding protein.

${ }^{*}$ Anaemia, $\mathrm{Hb}<110 \mathrm{~g} / \mathrm{l}$; Fe deficiency, ferritin $<12 \mu \mathrm{g} / \mathrm{l}$; Fe-deficient erythropoiesis, sTfR $>8.3 \mathrm{mg} / \mathrm{l} ;$ Fe-deficiency anaemia, ferritin $<12 \mu \mathrm{g} / \mathrm{l}$ and $\mathrm{Hb}<110 \mathrm{~g} / \mathrm{l}$; VAD, RBP $<0.7 \mu \mathrm{mol} / \mathrm{l}$.

tAdjusted for inflammation.

WRA in a cross-sectional survey in Côte d'Ivoire subsequent to the internal conflict of 2002-2007 but prior to the civil war in 2010.

From the demographic data, analysis showed that there were significant differences between urban and rural households in the construction of their houses and the availability of electricity. Hygiene is affected by environmental and living conditions, and in an affluent urban homes, it is possible that food can be stored and prepared in a way that minimizes the dangers of contamination because of the presence of a refrigerator, a cooking stove and tap water in the house. For the poorer rural households, where for the majority there is no refrigerator, food is cooked outside over a wood fire and water is carried from a central water pump, appropriate food hygiene is much more difficult to achieve. Personal hygiene is also more problematic in rural areas, where $56 \%$ of the respondents are defecating in the open and there is no convenient access to water to wash their hands. The consequence of these environmental conditions may be that vulnerable poorer households will be exposed to

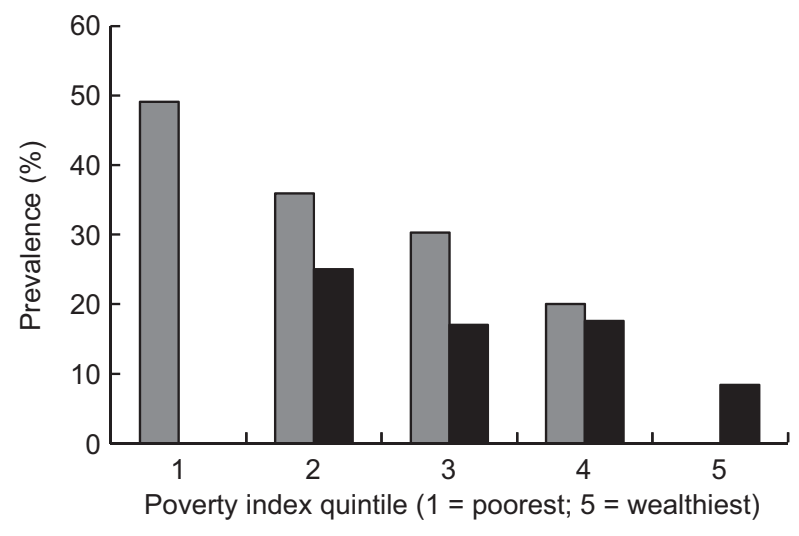

Fig. 2 Prevalence of Plasmodium spp. by poverty index and residency ( $\square$, rural; $\square$, urban) in pre-school children aged 6-59 months, Côte d'Ivoire, 2007

more pathogenic organisms than affluent households, which might lead to e.g. more diarrhoeal episodes in children. Although the effect of environmental conditions was not tested specifically in the present survey, there 
were significant differences in the prevalence of elevated CRP and AGP concentrations, which were higher in rural than urban pre-SAC, suggesting a greater exposure to inflammation in rural areas. Furthermore, others have found evidence that the presence of a latrine, especially in combination with access to clean water, has a positive effect in reducing the incidence of diarrhoea ${ }^{(29,30)}$.

\section{Pre-school age children}

Prevalence of stunting was $46 \%$, indicating a critical situation associated with chronic malnutrition, and the results suggest inappropriate infant and young child care practices and a lack of access to health facilities ${ }^{(31)}$. Wasting $(14 \cdot 1 \%)$ and underweight $(29 \cdot 0 \%)$ were also at worrying levels ${ }^{(32)}$. In comparison with data from a survey in Côte d'Ivoire in 2003/04, where the prevalence of stunting in rural pre-SAC was $27 \cdot 7 \%$ (cf. $46 \cdot 2 \%$ in 2007)

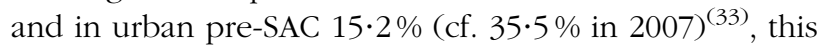
represents a substantial increase in chronic malnutrition, probably due to the poor food security situation and collapse of the economy due to politico-military conflict. However, the most striking differences were in the prevalence of severe stunting and underweight $(\mathrm{HAZ}<-3$ and $\mathrm{WAZ}<-3$, respectively) in rural pre-SAC, which was twice that of the urban children. Anthropometric indicator Z-scores below -3 are associated with increased risk to health: based on WHO data, such children have nine times the risk of death than a child with a $\mathrm{WHZ}>-1^{(34)}$. Further, data from Deen et al. ${ }^{(35)}$ suggest that stunted children experience more episodes of malaria than those who are not stunted (relative risk $=1 \cdot 35 ; 95 \%$ CI $1 \cdot 08$, 1.69). However, the authors found malaria had no further detrimental effect on nutritional status across a malarial season, suggesting that chronically malnourished children were at higher risk for developing malaria episodes. The inference from their study is that reducing the prevalence of stunting may be of great value in reducing the number of episodes and, therefore, the impact of malaria in young children.

The prevalence of Plasmodium spp. parasitaemia and of elevated acute-phase proteins was $2 \cdot 7$ and 1.7 times higher, respectively, in rural than urban children. The presence of inflammation, as identified by elevated CRP and/or AGP, had a significant effect on the plasma concentrations of ferritin, which were higher in children in rural areas, even after correction using the meta-analysis method of Thurnham et al. ${ }^{(26)}$. Furthermore, children with inflammation and Plasmodium parasites had mean ferritin concentrations that were $12 \mu \mathrm{g} / \mathrm{l}$ higher than those of children without Plasmodium parasites, even after adjustment, suggesting an additional detrimental effect of Plasmodium parasites on ferritin concentrations. Therefore in malaria-endemic areas, correction of ferritin concentrations in pre-SAC using the Thurnham meta-analysis method may not be enough and this will need to be investigated further.
Regardless of residency, anaemia prevalence was higher than the WHO threshold, classifying anaemia as a severe public health problem in Côte d'Ivoire ${ }^{(8)}$. It is commonly assumed that about half of anaemia is due to Fe deficiency ${ }^{\left({ }^{36)}\right.}$ but in the present survey, a much smaller proportion of the anaemia coincided with Fe-deficiency anaemia, especially in the rural children. To understand the aetiology of the anaemia in this population is difficult but it is probably multifactorial: resulting from Fe, vitamin A and folate deficiencies, haemoglobinopathies and/or the presence of Plasmodium parasites. Haemoglobinopathies were identified in only a small percentage of a Côte d'Ivoire population in a study by StaubliAsobayire $^{(11)}$ (sickle-cell trait 9\%, Hb-C 9\%, all other haemoglobinopathies $<1 \%$ ) and were not tested in the present survey. Folate is essential in haematopoiesis, and where it is limiting in the diet megaloblastic anaemia can develop. However, we did not measure red cell width or folate concentrations in the pre-SAC, but can speculate that it was present, as folate deficiency was found in $86 \%$ of the WRA.

Anaemia was present in $76.0 \%$ of rural and $68.2 \%$ of urban children, but Fe deficiency was found in only $8.6 \%$ of rural compared with $22 \cdot 1 \%$ of urban children. Because of the difference in exposure to inflammation and Plasmodium parasites, it is likely that the anaemia in the rural children was due to a larger extent to inflammation rather than Fe deficiency. This hypothesis is further supported when considering the percentage of children with $\mathrm{Fe}$ deficient erythropoiesis; in the rural children only $7 \cdot 0 \%$ had concentrations $\geq 8.3 \mathrm{mg} / \mathrm{l}$ but in urban children the percentage was $15 \cdot 3 \%$. The WHO/CDC Consultative Group $^{(25)}$ in 2007 proposed that where the percentage of Fe deficiency (defined by low ferritin concentration) in a population group is $\geq 20 \%$ and the percentage with Fedeficient erythropoiesis is $\geq 10 \%$, then Fe deficiency is prevalent. Conversely, when the percentage of Fe deficiency is $<10 \%$ and of Fe-deficient erythropoiesis is $<20 \%$, then Fe deficiency is not present. Thus the data presented herein suggest that Fe deficiency was present in the urban children but not in the rural children. Therefore, the likely mechanism whereby inflammation is contributing to anaemia in the rural children is by the production of inflammatory cytokines, such as IL-6, on a key mediator of hypoferraemia in inflammation, hepcidin, which can suppress both haematopoiesis by inhibition of erythropoietin production and erythropoiesis by inhibition of Fe mobilization. In addition, the presence of Plasmodium parasites $38.7 \%$ prevalence in rural children) can result in haemolysis, when potentially tissuedamaging $\mathrm{Hb}$ is released into the plasma and is bound by the protective acute-phase protein, haptoglobin ${ }^{(37)}$. The haptoglobin-Hb complex is rapidly cleared from the plasma on circulating macrophages. The Fe-loaded macrophages migrate to the reticulo-endothelial system where they can lodge for long periods, making the 
Fe unavailable until the inflammatory response subsides and the Fe component can be recycled for erythropoiesis.

Concentrations of sTfR can be stimulated by a number of factors, e.g. cytokines ${ }^{(25)}$, and so have to be interpreted with caution. The relationship between Fe status and STfR concentrations in anaemia is inversely dependent on the degree of inflammation, since inflammation will depress erythropoietin production and marrow activity. However, the cyclical effect of Plasmodium parasites on disrupting red cell metabolism may be associated with sudden changes in Fe status, which can stimulate or depress erythropoiesis. Verhoef et al. found that concentrations of STfR were increased in infants with both Fe-deficiency anaemia and malaria, but that there was a significantly greater increase in concentrations of STfR in those with anaemia and malaria ${ }^{(38)}$. In the present survey, children with Fe deficiency had significantly higher sTfR concentrations than those without, and STfR concentrations were significantly higher in children with both anaemia and Plasmodium parasites compared with those who had neither, suggesting an increase in sTfR concentrations was an attempt to increase erythropoiesis.

VAD is a severe public health problem nationally in Côte d'Ivoire, but there were differences in VAD among pre-SAC by eco-region; those in the central (13.0\%) and south-eastern (12.4\%) regions had a lower prevalence. Apart from the impact of VAD on the health of the child, significantly increasing the risk of severe illness from common childhood infections such as diarrhoeal disease and measles, vitamin A also appears to influence Fe metabolism but the precise mechanism is not yet elucidated ${ }^{(39)}$.

\section{Women of reproductive age}

The majority of women were of normal BMI, with $<5 \%$ obese and $<12 \%$ underweight, but there were urban/ rural differences as urban women had higher BMI and weight, but not height, than those in rural areas. There was a lower prevalence of Plasmodium parasites and a lighter parasite load in women than children, although there were similar rural/urban differences.

Anaemia was a severe public health problem in the population $^{(8)}$ and there were significant urban/rural and eco-regional differences, with those in the rural, south, south-east and northern areas being worst affected. Whether the internal conflict was an underlying reason is hard to say, although most conflict events between 2002 and 2007 took place in the south, the central-west and the north of the country ${ }^{(14)}$.

Ferritin concentrations were in the normal range, again with significant urban/rural differences, but as with the children, there were more WRA with Fe deficiency in urban than rural areas. Although the prevalence of Plasmodium parasites was lower in the women than in the children, there was still a rural/urban difference with rural women having more parasitaemia, which could be the underlying reason for the higher ferritin concentrations in those women. There were no significant differences in the prevalence of Fe-deficient erythropoiesis or Fe-deficiency anaemia between the rural and urban women.

Mean folate concentrations were below the accepted cut-off $^{(24)}$. Folate deficiency was high and differed significantly between rural and urban populations, and among the eco-regions. Fresh green vegetables are a good source of natural folate but losses during harvesting, storage and cooking can be high, as can losses from cooking animal products, while staples such as rice and wheat are low in folate and may contribute to poor folate status ${ }^{(40)}$. In contrast, vitamin $\mathrm{B}_{12}$ status was good, with mean values above the recommended cut-off ${ }^{(24)}$, although there was a difference in prevalence of deficiency between the rural $(24 \cdot 2 \%)$ and urban areas $(12 \cdot 8 \%)$. The rural/urban difference may be due to differences in dietary intake of vitamin $\mathrm{B}_{12}$, which might be lower in the rural communities, but without dietary intake data it is impossible to know. Vitamin $B_{12}$ is produced by bacteria and is naturally occurring in animal products. However, contamination of other non-animal foods with bacteria may be a possible source of vitamin $\mathrm{B}_{12}$ for the rural populations, where animal foods may be less affordable ${ }^{(41)}$.

Mean RBP concentrations were good, although lower in the rural population. There was very little VAD and there were no differences in VAD prevalence among the eco-regions or between rural and urban areas.

\section{Conclusions}

The micronutrient interventions under consideration by the Government of Côte d'Ivoire include the fortification of the staple wheat flour with Fe and folic acid, and an improvement in the coverage of vegetable oil fortified with vitamin A. Folate deficiency was present in $86 \%$ of WRA, indicating that fortification of flour with folic acid could be an effective public health policy, particularly for WRA with market access to fortified products. VAD was not prevalent among WRA and therefore fortified vegetable oil is not a priority for WRA, but it could be of benefit to pre-SAC, especially the older children eating complementary or family foods. Younger children are dependent on breast milk and as the women's vitamin A status is good, breast milk vitamin A concentrations should also be adequate. Lower vitamin A concentrations seen in the children in the survey were most likely due to inflammation and especially the presence of Plasmodium parasites.

\section{Acknowledgements}

Sources of funding: The Global Alliance for Improved Nutrition (GAIN), UNICEF, Helen Keller International (HKI), the Swiss Federal Institute of Technology (ETH Zurich), the Institut National de Santé Publique en Côte 
d'Ivoire (INSP), Map International, the Centre Suisse des Recherches Scientifiques and Unilever provided financial, technical or in-kind contributions to this study, a support that is gratefully acknowledged; none of the commercial funders had any role in the design, analysis or writing of this article. Conflicts of interest: None of the authors have a conflict of interest to declare. Authors' contributions: F.R., A.B.T., V.K.-G., P.E.B. and M.B.Z. designed the study; F.R., A.B.T., V.K.-G., P.E.B., J.G.E. and M.B. conducted the research; F.R., C.N.-C., A.B.T. and C.G.N.M.-T. analysed the data; F.R., C.N.-C. and C.G.N.M.-T. wrote the first draft of the manuscript; all authors contributed to, read and approved the final manuscript.

\section{References}

1. McLean E, Cogswell M, Egli I et al. (2009) Worldwide prevalence of anaemia, WHO Vitamin and Mineral Nutrition Information System, 1993-2005. Public Health Nutr 12, 444-454.

2. Crawley J (2004) Reducing the burden of anemia in infants and young children in malaria-endemic countries of Africa: from evidence to action. Am J Trop Med Hyg 71, 25-34.

3. Yip R \& Dallman PR (1988) The roles of inflammation and iron deficiency as causes of anemia. Am J Clin Nutr $\mathbf{4 8}$, $1295-1300$

4. Suharno D, West CE, Muhilal et al. (1993) Supplementation with vitamin $\mathrm{A}$ and iron for nutritional anaemia in pregnant women in West Java, Indonesia. Lancet $\mathbf{3 4 2}$, $1325-1328$.

5. Gilgen D \& Mascie-Taylor CG (2001) The effect of anthelmintic treatment on helminth infection and anaemia. Parasitology 122, 105-110.

6. Boccio JR \& Iyengar V (2003) Iron deficiency: causes, consequences, and strategies to overcome this nutritional problem. Biol Trace Elem Res 94, 1-32.

7. Hurrell RF (2002) Fortification: overcoming technical and practical barriers. J Nutr 132, 4 Suppl., 806S-812S.

8. World Health Organization/UNICEF/United Nations University (2001) Iron Deficiency Anaemia: Assessment, Prevention and Control. A Guide for Programme Managers, WHO/NHD/01.3. Geneva: WHO.

9. Staubli Asobayire F, Adou P, Davidsson L et al. (2001) Prevalence of iron deficiency with and without concurrent anemia in population groups with high prevalences of malaria and other infections: a study in Cote d'Ivoire. Am J Clin Nutr 74, 776-782.

10. Aguayo VM \& Baker SK (2005) Vitamin A deficiency and child survival in sub-Saharan Africa: a reappraisal of challenges and opportunities. Food Nutr Bull 26, 348-355.

11. Staubli-Asobayire F (2000) Development of a food fortification strategy to combat iron deficiency in the Ivory Coast. Thesis no. 13730, ETH Zürich.

12. Thurnham DI, McCabe GP, Northrop-Clewes CA et al (2003) Effects of subclinical infection on plasma retinol concentrations and assessment of prevalence of vitamin A deficiency: meta-analysis. Lancet 362, 2052-2058.

13. World Health Organization/UNICEF (2005) GIVS Global Immunisation Vision and Strategy 2006-2015. Geneva/ New York: WHO/UNICEF; available at http://whqlibdoc. who.int/hq/2005/WHO_IVB_05.05.pdf

14. Minoiu C \& Shemyakina ON 2012. Armed conflict, household victimization and child health in Cote d'Ivoire. http:// www.ecineq.org/milano/WP/ECINEQ2012-245.pdf (accessed October 2012)
15. Rohner F, Tschannen AB, Northrop-Clewes C et al. (2012) Comparison of a possession score and a poverty index in predicting anaemia and undernutrition in pre-school children and women of reproductive age in rural and urban Cote d'Ivoire. Public Health Nutr 15, 1620-1629.

16. Institut National de la Statistique and UNICEF (2006) Côte d'Ivoire, 2006 - Multiple Indicator Cluster Survey 3. Abidjan: Côte d'Ivoire; available at http://www.childinfo.org/mics3_surveys.html

17. Kish L (1949) A procedure for objective respondent selection within the household. J Am Stat Assoc 44, 380-387.

18. World Health Organization (1990) Diagnosis of Malaria. PAHO Scientific Publication Series no. 512. Geneva: WHO.

19. Erhardt JG, Estes JE, Pfeiffer CM et al. (2004) Combined measurement of ferritin, soluble transferrin receptor, retinol binding protein, and C-reactive protein by an inexpensive, sensitive, and simple sandwich enzyme-linked immunosorbent assay technique. J Nutr 134, 3127-3132.

20. Horne DW \& Patterson D (1988) Lactobacillus casei microbiological assay of folic acid derivatives in 96-well microtiter plates. Clin Chem 34, 2357-2359.

21. Tabachnick BG \& Fidell LS (2007) Using Multivariate Statistics. Boston, MA: Pearson/Allyn \& Bacon.

22. Gwatkin DR, Rustein S, Johnson K et al. (2000) Socioeconomic Differences in Health, Nutrition and Population in the Côte d'Ivoire. Washington, DC: World Bank.

23. Shetty PS \& James WP (1994) Body Mass Index. A Measure of Chronic Energy Deficiency in Adults. FAO Food and Nutrition Paper no. 56. Rome: FAO.

24. Allen LH, de Benoist B, Dary O et al. (2006) Guidelines on Food Fortification with Micronutrients. Geneva: WHO.

25. World Health Organization/Centers for Disease Control and Prevention (2007) Assessing the Iron Status of Populations: Including Literature Reviews: Report of a Joint World Health Organization/Centers for Disease Control and Prevention Technical Consultation on the Assessment of Iron Status at the Population Level. Geneva: WHO.

26. Thurnham DI, McCabe LD, Haldar S et al. (2010) Adjusting plasma ferritin concentrations to remove the effects of subclinical inflammation in the assessment of iron deficiency: a meta-analysis. Am J Clin Nutr 92, 546-555.

27. World Health Organization (1996) Indicators for Assessing Vitamin A Deficiency and Their Application in Monitoring and Evaluating Intervention Programmes, WHO/NUT/ 96.10. Geneva: WHO.

28. World Health Organization (2009) Global Prevalence of Vitamin A deficiency in Populations at Risk, 1995-2005. WHO Global Database on Vitamin A Deficiency. Geneva: WHO.

29. Root GP (2001) Sanitation, community environments, and childhood diarrhoea in rural Zimbabwe. J Health Popul Nutr 19, 73-82.

30. Esrey SA, Habicht JP \& Casella G (1992) The complementary effect of latrines and increased water usage on the growth of infants in rural Lesotho. Am J Epidemiol 135, 659-666.

31. UNICEF (2009) Fact sheet: Nutrition Survey Cote d'Ivoire. N.1/SURVIE/NOVEMBRE 2009. http://www.unicef.org/ cotedivoire/Nutritionfactsheet-_EN.pdf (accessed October 2012).

32. World Health Organization (2012) Global Database on Child Growth and Malnutrition. http://www.who.int/ nutgrowthdb/about/introduction/en/index $5 . h t m l$ (accessed October 2012).

33. United Nations System Standing Committee on Nutrition (2005) Nutrition in Crisis Situation, Vol 6, May 2005 - Ivory Coast. http://www.unsystem.org/scn/archives/nics06/index. htm\#Ivory\%20Coast (accessed August 2013). 
34. World Health Organization/UNICEF (2009) WHO child growth standards and the identification of severe acute malnutrition in infants and children. http://www.who.int/nutrition/publications/ severemalnutrition/9789241598163_eng.pdf (accessed August 2013).

35. Deen JL, Walraven GE \& von Seidlein L (2002) Increased risk for malaria in chronically malnourished children under 5 years of age in rural Gambia. J Trop Pediatr $\mathbf{4 8}$, 78-83.

36. World Health Organization (2012) Priorities in the assessment of vitamin A and iron status in populations, Panama City, Panama, 15-17 September 2010. http://apps.who. int/iris/bitstream/10665/75334/1/9789241504225_eng.pdf (accessed October 2012).
37. Weiss $G$ (2009) Iron metabolism in the anemia of chronic disease. Biochim Biophys Acta 1790, 682-693.

38. Verhoef H, West CE, Kraaijenhagen R et al. (2002) Malarial anemia leads to adequately increased erythropoiesis in asymptomatic Kenyan children. Blood 100, 3489-3494.

39. Semba RD \& Bloem MW (2002) The anemia of vitamin A deficiency: epidemiology and pathogenesis. Eur J Clin Nutr 56, 271-281.

40. Food and Agricultural Organization of the United Nations/ World Health Organization (2001) Human Vitamin and Mineral Requirements. Report of a Joint FAO/WHO Expert Consultation, Bangkok, Thailand. Rome: FAO.

41. Herbert V (1988) Vitamin B-12: plant sources, requirements, and assay. Am J Clin Nutr 48, 852-858. 